\title{
Biodegradation of Diethylketone by Penicillium sp. and Alternaria sp. - A Comparative Study Biodegradation of Diethylketone by Fungi
}

\author{
Filomena Costa*, Marta Neto, Ana Nicolau and Teresa Tavares
}

Centre of Biological Engineering, University of Minho, Campus de Gualtar 4710-057, Braga, Portugal

\begin{abstract}
Two contaminating fungi were isolated from a bioreactor containing diethylketone and Streptococcus equisimilis, subsequently characterized at molecular level and identified as belonging to the Alternaria and Penicillium genera. The ability of these fungi to biodegrade DEK is evaluated. The kinetic parameters are estimated using four growth kinetic models for biodegradation of organic compounds available in literature. The experimental data for Alternaria sp. and Penicillium sp. was found to be better fitted by the Haldane and the Luong respectively. Biodegradation rate kinetics was evaluated using zero-order, pseudo-first order, pseudo-second order and three-half order models. The pseudo-second-order model was found suitable for all the concentrations of DEK tested for the biodeg-

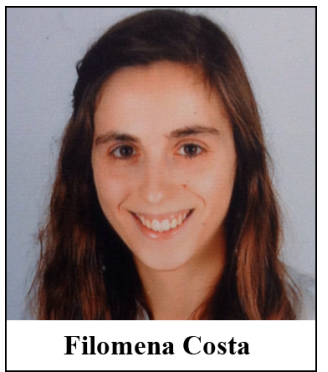
radation assays using Penicillium sp. whereas for the Alternaria sp. this model just describes properly the assays with initial concentrations of DEK higher than $0.5 \mathrm{~g} / \mathrm{L}$. The percentage of biodegraded DEK were approximately $100 \%$, for all the initial concentrations tested.
\end{abstract}

Keywords: Biodegradation, diethylketone, fungi, genetic identification, kinetics.

\section{INTRODUCTION}

Ketones are important trace constituents of the Earth's troposphere and like other volatile organic compounds (VOCs), they are emitted into the atmosphere from natural and anthropogenic sources. Ketones are commonly employed in the manufacturing of pharmaceuticals, plastics, paints, rubbers and lubrificants and are released by the petrol and petrochemical industries into the environment $[1,2]$. The widespread usage of these compounds has resulted in their increasing release and accumulation in various types of water bodies and locations in the environment [2-5]. Among the several ketones used, diethylketone (DEK) stands out, not only for its intensive usage in several anthropogenic activities as solvent or polymer precursor in industries [6], as an intermediate in the synthesis of pharmaceuticals, flavours and pesticides [2], but also because it reacts with $\mathrm{OH}$ radicals promoting the formation of ozone and other components of the photochemical smog in urban areas [6], its persistence in water, soil and air and its high mobility (www.cdc.gov) and ability to form toxic and phototoxic intermediates [7]. Diethylketone inhalation may cause irritation in the eyes, skin, mucous membrane, cough and sneezing according to the Occupational Safety and Health hazards (OSHA) of United States Department Labor. Prolonged exposure results in tachycardia, nausea, shortness of breath, dizziness, fainting, coma and even death. Diethylketone does not bind well to soil and thus pollutes groundwater. It has acute and chronic toxicity to the aquatic life as well.

Biological treatments techniques such as biodegradation are an attractive approach for decontamination of wastewater

*Address correspondence to this author at the Centre of Biological Engineering, University of Minho, Campus de Gualtar 4710-057, Braga, Portugal; Tel: +351 253601 976; Fax: +351 253604 429;

E-mail: filomenacpcosta@ceb.uminho.pt from VOCs such as DEK as compared with some conventional techniques such as adsorption on granular activated carbon (GAC), air-stripping, oxidation, with or without flame, thermal degradation [7], condensation and incineration [8]. The main disadvantages of these conventional techniques are the emission of other gases $\left(\mathrm{NO}_{\mathrm{x}}\right)$, which requires additional costs for the secondary treatment, generation of contaminated solid waste, low efficiency, excessive use of chemicals, too expensive from operational, maintenance and equipment perspectives [7,9]. Studies conducted by different authors demonstrated that biological treatments can replace the conventional techniques and involve lower investment, in terms of capital and operational costs, show a good operational stability and are environmental friendly as they lead to the formation of less and/or non-dangerous products [10-16]. The biodegradation process of hazardous compounds by microorganisms is extremely complex and may result from the combination of several factors such enzymatic degradation, binding by extracellular polymeric substances (EPS) or binding by functional groups present on the cell wall. The use of fungi to degrade complex and persistent natural materials and hazardous compounds is well known [17-19]. The cell wall and extracellular exudates of fungi exhibit a variety of Lewis-acid functional groups including carboxyl, phosphoryl, amine, hydroxyl moieties and sulphates, most of which have a strong binding affinity for cationic aqueous species [18]. The major structural components of fungal cell walls are chitin and chitosan, comprised of polymerized Nacetyl-D-glucosamine and D-glucosamine, respectively, along with a variety of extracellular polymers including $\alpha$ and $\beta$-glucans, cellulose and a diversity of mannoproteins and glycoproteins [18], which have been implicated in the sequestering of metals [20]. Fungi are able to produce different enzymes including pectinolytic and amylolytic systems, invertases, cellulases and hemicelullases, pectinases, prote- 
ases, laccases, phytases, $\alpha$-glucuronidases, mannanases and lipases which are involved in the degradation of hazardous compounds [21]. Atagana [17] used indigenous soil fungi present in contaminated soil with creosote to biodegrade phenol, $o$-cresol, $m$-cresol and $p$-cresol. These authors reached degradation yields between 84 and $100 \%$ for the phenols tested. Chai et al. [22] investigated the biodegradation of 2,2,-bis (4- hydroxyphenyl) propane by 26 fungi belonging to different groups: Aspergilllus, Fusarium, Penicillium, Fungi imperfecti, Ascomycetes and Zygomycetes. Among the 26 strains tested, 11 reached biodegradation percentages equal or higher than $50 \%$. Pipíška et al. [23] investigated the sorption of cobalt by the foliose lichen $\mathrm{Hy}$ pogymnia physodes from $\mathrm{CoCl}_{2}$ solutions spiked with ${ }^{60} \mathrm{Co}^{2+}$. The maximum uptake value was reached within 1 hour and these authors were also able to conclude that after 24 hours assays, the biosorption is not $\mathrm{pH}$-dependent within the range of $\mathrm{pH} 4-7$, negligible at $\mathrm{pH} 2$ and is not dependent on metabolic activity.

This work aims the development of an environmental technology applicable to the treatment of aqueous solution contaminated with DEK and is focused on the detailed kinetic study for the biodegradation of DEK. The effect of initial concentration of DEK $(0.5 \mathrm{~g} / \mathrm{L}$ to $4 \mathrm{~g} / \mathrm{L})$ on the growth of the fungi and on the biodegradation process was evaluated. The information collected from these experimental studies was used to calculate the growth kinetic constants and the degradation kinetic constants from different models, Monod [24], Powell [25], Haldane [26], Luong [27] and Edwards [28], reported in literature as models with application in biodegradation of organic pollutants. The intensive modelling effort described herein will allow the definition of general equations to be applied to many other contaminated systems foreseeing the overall efficiency of the biosorption process while still in the project phase. The importance of this work is notorious because not only it defends a quite straightforward technology to biodegrade high concentrations of DEK from contaminated aqueous solutions using microorganisms, but also because up to the present knowledge these two fungi were never used to biodegrade DEK.

\section{MATERIAL AND METHODS}

\subsection{Organisms, Culture Media and Chemicals}

The appearance of successive fungi contaminations on different stages of the experiments, in bioreactors employed for the treatment of aqueous solutions with DEK (1-7.5 g/L) using S. equisimilis, promoted the formulation of the hypothesis that those microorganisms could be able to biodegrade DEK or, at least, their growth would not be inhibited by DEK. In order to test this hypothesis, a sample from the contaminated bioreactor was collected and subsequently inoculated in a selective culture media to allow the growth of fungi and inhibit the bacterial growth. For this purpose Dichloran Rose Bengal Chloramphenicol agar (DRBC) with composition described by Ayala et al. [21] were used for screening and isolation while Malt Extract Agar (MEA) media with composition also described by those authors was used for the maintenance of the two fungi. Brain Heart Infusion (OXOID CM1135) culture medium with the following composition: brain infusion solids $(12.5 \mathrm{~g} / \mathrm{L})$, beef heart in- fusion solids $(5.0 \mathrm{~g} / \mathrm{L})$, proteose peptone $(10.0 \mathrm{~g} / \mathrm{L})$, glucose $(2.0 \mathrm{~g} / \mathrm{L})$, sodium chloride $(5.0 \mathrm{~g} / \mathrm{L})$ and di-sodium phosphate $(2.0 \mathrm{~g} / \mathrm{L})$,was used for the fungal growth and concentration. Diethylketone was purchased from Acros Organics (98\% pure) and it was diluted in sterilized distilled water.

\subsection{Isolation of Fungi from Contaminated Bioreactors}

A sample of the contaminated bioreactors was aseptically collected and spread directly in a DRBC agar medium, and maintained in the dark, at $37^{\circ} \mathrm{C}$ for 5 days. The colonies formed were aseptically collected and successively subcultured in sterilized DRBC agar medium. This procedure was repeated tenfold in order to ensure the isolation of the culture.

The cultures of each fungal isolate were transferred, subcultured aseptically in MEA agar medium at $25^{\circ} \mathrm{C}$ for 5 days and subsequently stored at $-2^{\circ} \mathrm{C}$.

\subsection{Extraction of Fungal DNA}

Fungal isolates were inoculated separately into a new fresh MEA liquid medium and maintained at $25 \pm 2{ }^{\circ} \mathrm{C}, 150$ rpm for 24 hours. Samples of $2 \mathrm{~mL}$ were collected and centrifuged at $13400 \mathrm{rpm}$ for 10 minutes (Eppendorf MiniSpin 9056, F-45-12-11). DNA extraction was performed according to PowerSoil ${ }^{\circledR}$ Dna Isolation Kit, MO Bio Laboratories, Inc.

\subsection{Molecular Identification of the Two Fungi}

DNA extract was used to amplify the Internal Transcribe Sequences (ITS) surrounding the 5.8S-coding sequence, situated between the Small SubUnit-coding sequence (SSU) and the Large SubUnit-coding sequence (LSU) of the ribosomal operon. The ITS region was amplified by PCR using fungal primers ITS1-F 5'-CTTGGTCATTTAGAGGAAG TAA-3' and ITS4 5'-TCCTCCGCTTATTGATATGC-3'. PCR reactions $(50 \mu \mathrm{L})$ comprised $1 \mu \mathrm{L}$ of genomic DNA, $35.25 \mu \mathrm{L}$ of autoclaved ultra-pure water (Millipore Milli-Q Synthesis, 1998), $5 \mu \mathrm{L}$ of PCR buffer (10xPCR Rxn Buffer, Invitrogen ${ }^{\mathrm{TM}}$ Life Technologies), $3 \mu \mathrm{L}$ of $50 \mathrm{mM} \mathrm{MgCl}_{2}$ (Invitrogen $^{\mathrm{TM}}$ Life Technologies), $1 \mu \mathrm{L}$ of dNTP Mix, $1 \mu \mathrm{L}$ of forward primer ITS1-F, $1 \mu \mathrm{L}$ of reverse primer ITS 4 (Invitrogen $^{\mathrm{TM}}$ Life Technologies), $0.25 \mu \mathrm{L}$ of $500 \mathrm{U}$ Taq DNA polymerase Recombinant (Invitrogen ${ }^{\mathrm{TM}}$ Life Technologies), $2.5 \mu \mathrm{L}$ of $1 \% \mathrm{~W}-1$ solution (Invitrogen ${ }^{\mathrm{TM}}$ Life Technologies). Amplifications were carried out in a Bio-Rad MYCYCLER thermal cycler using a temperature gradient protocol as follows: initial denaturation at $95^{\circ} \mathrm{C}$ for 3 minutes, followed by 39 cycles of denaturation at $95^{\circ} \mathrm{C}$ for 30 seconds, annealing at $50.7^{\circ} \mathrm{C}$ for 30 seconds, extension at $72^{\circ} \mathrm{C}$ for 36 seconds and final extension $72^{\circ} \mathrm{C}$ for 5 minutes. PCR amplification products were analyzed by electrophoresis and subsequently purified according to the PCR Clean-up Gel extraction, NucleoSpin ${ }^{\oplus}$ Extract II kit. The sequencing of the PCR purified products were conducted by Eurofins MWG Operon (Ebersberg, Germany) and subjected to a GenBank BLAST in the National Center for Biotechnology Information (NCBI database) search to retrieve sequences of closely related taxa. 


\subsection{Biodegradation Experiments}

Each fungal isolate was grown for 24 hours at $37^{\circ} \mathrm{C}$ and $150 \mathrm{rpm}$ in $500 \mathrm{~mL}$ of a BHI culture medium with the composition described in 2.1. Three sets of $150 \mathrm{~mL}$ of this inoculated medium were transferred to 3 new BHI culture media $(1000 \mathrm{~mL})$. These cultures were grown for 48 hours at $37^{\circ} \mathrm{C}$ and $150 \mathrm{rpm}$. After this period, the biomass was recovered on a Sigma 4K15 centrifuge (RCF of 7950) and the supernatant was collected on a sterile bottle for later use. The biomass pellets were re-suspended on a smaller volume of the collected medium, being this volume calculated so that a final biomass concentration in culture between 2 and $3 g_{\text {bio- }}$ mass $/ \mathrm{L}[7]$ might be obtained.

The experiments were conducted in 250-mL Erlenmeyer flasks containing $150 \mathrm{~mL}$ of DEK solution which were then inoculated with the previous concentrated biomass. The diethylketone concentrations used for these assays were on the range $0.5-4 \mathrm{~g} / \mathrm{L}$. A sample was taken at different time intervals, centrifuged at $13400 \mathrm{rpm}$ for 10 minutes and the optically density (OD) was measured. The supernatant was used to quantify the concentration of DEK. The assays were conducted in duplicate, during 5-7 days, at $37^{\circ} \mathrm{C}$ and $150 \mathrm{rpm}$ and the results are an average of both duplicates. The relative standard deviation and relative error of the experimental measurements were less than $2 \%$ and $5 \%$, respectively.

\subsection{Analytical Methods}

\subsubsection{Cell Growth Determination}

Cell density was monitored spectrophotometrically (PG Instruments T60-UV Visible Spectrophotometer) by measuring the optical density at $\lambda=620 \mathrm{~nm}$. The OD was then converted to biomass concentration using the following equations:

For the Penicillium sp. fungi,

$B C=1.9625 \mathrm{OD}_{620 \mathrm{~nm}}$

For the Alternaria sp. fungi,

$B C=2.0792 \mathrm{OD}_{620 \mathrm{~nm}}$

\subsubsection{Quantification of DEK Concentration}

Gas chromatography (GC) analyses were performed in order to evaluate the biodegradation capacity of these fungi through the decreasing of diethylketone concentration over time. The GC employed herein was a GC-MS Varian 4000, equipped with a flame ionization detector (FID) and mass spectrometry (MS). The separations were performed using a
Meta Wax column $(30 \mathrm{~m} \times 0.25 \mathrm{~mm} \times 0.25 \mu \mathrm{m})$. The operating conditions were as follows: the column was held initially at a temperature $50^{\circ} \mathrm{C}$, then heated at $10^{\circ} \mathrm{C} /$ minute to $100^{\circ} \mathrm{C}$, held at $100^{\circ} \mathrm{C}$ for 4 minutes, then heated again at $40^{\circ} \mathrm{C} /$ minute to $200^{\circ} \mathrm{C}$ and finally held at $200^{\circ} \mathrm{C}$ for $2 \mathrm{~min}-$ utes. The temperature of injector and detector were maintained at $250^{\circ} \mathrm{C}$. Nitrogen was used as a carrier gas at a flow rate of $30 \mathrm{~mL} /$ minute and the injections were performed in the split mode with a split ratio of 1:7. Under these conditions, the retention time for diethylketone was 3.2 minutes.

\subsection{Growth Kinetics of Fungi}

In the present study, the linear and nonlinear growth kinetic models were fitted by linear and nonlinear least squares methods using MATLAB software. The models used were Monod [24], Powell [25], Haldane [26], Luong [27] and Edwards [28].

\subsection{Diethylketone Biodegradation Kinetics}

The experimental biodegradation data were modelled using the linearized form of the zero order, pseudo-first, pseudo second and three-half order equations [29-31].

\section{RESULTS AND DISCUSSION}

\subsection{Isolation and Genetic Identification of DEK Degrad- ing Fungi}

Two fungi (one green and the other white) were collected from the contaminated bioreactors working with aqueous solutions of DEK and S. equisimilis, according to the methods described in 2.2, and differentiated by both macroscopic characteristics when cultured in agar plates (colour, shape, texture) and microscopic ones (cells morphology and Gram staining). The main characteristics of these two fungi are listed in (Table 1). The green and white fungi were identified by sequencing the ITS region and possess high homology respectively with the Alternaria genera (99\%, accession number (KC623563.1) and with the Penicillium genera (99\%, accession number (HQ850362.1). According to Agathos et al. [10], the appearance and abundance of fungi in the bioreactors is related to the acidic conditions inside the reactor and to their broader tolerance to changes in $\mathrm{pH}$ values, concomitant with ketones degradation.

\subsection{Degradation of DEK by the Two Fungi}

In the present study, concentrated biomass of the isolated fungi and initial concentrations of DEK of $0.5,1,2$ and $4 \mathrm{~g} / \mathrm{L}$

Table 1. Morphological characteristics: growth, front view, back view, characteristics of hyphae and Gram staining of the two fungi.

\begin{tabular}{|c|c|c|c|c|c|}
\hline Fungi & Growth & Front View & Back View & Character of Hyphae \\
\hline \hline White & Rapid & White with central yellow green powdery appearance & White & $\begin{array}{c}\text { Irregular form, with raised elevation } \\
\text { and undulate margins }\end{array}$ & Gram negative \\
\hline Green & Rapid & Olive green with wrinkled appearance & Dark green & $\begin{array}{c}\text { Irregular form, with umbonate eleva- } \\
\text { tion and entire margins }\end{array}$ \\
\hline
\end{tabular}


were used. Diethylketone was supplied as the only carbon and energy source. The biomass concentration for each isolated fungi was calculated using a calibration curve (optical density versus biomass concentration, equations 1 and 2) and the measurement of the dry weight. The effect of the initial concentration of diethylketone on the growth of the biomass used and on the biodegradation process was evaluated.

In (Table 2) the maximum concentration of biomass and the specific growth rate for each initial concentration of diethylketone, for Penicillium sp. and for Alternaria sp. are listed. It is possible to observe from (Table 2) that the maximum biomass concentration and the maximum specific growth rate of Penicillium sp. $\left(0.582 \mathrm{~g} / \mathrm{L}\right.$ and $9.0 \times 10^{-4} \mathrm{~h}^{-1}$, respectively) are obtained for an initial concentration of DEK of $1 \mathrm{~g} / \mathrm{L}$ and $2 \mathrm{~g} / \mathrm{L}$ respectively. For higher concentrations these values start to slightly decrease. For Alternaria sp. the highest value of biomass concentration and of specific growth rate $\left(1.065 \mathrm{~g} / \mathrm{L}\right.$ and $1.9 \times 10^{-3} \mathrm{~h}^{-1}$, respectively) are obtained for an initial concentration of DEK of $0.5 \mathrm{~g} / \mathrm{L}$. For higher concentrations of DEK the biomass concentration and the specific growth rate decrease significantly suggesting that initial concentration of DEK higher than $0.5 \mathrm{~g} / \mathrm{L}$ negatively affects the microbial growth.

\subsubsection{Biodegradation of DEK by the Penicillium sp.}

(Fig. 1) shows the time profile of Penicillium sp. growth and the biodegradation percentage (\%) of DEK as a function of time, for all the initial concentrations tested using the Penicillium sp. as biodegradation agent. The biodegradation of DEK is fast in the first 80 hours, after that it slows down until values equal and higher than $99 \%$ are reached (for initial concentrations higher than $0.5 \mathrm{~g} / \mathrm{L}$ ). This initial input is explained by the availability of the biomass and the need of the biomass to consume nutrients such as carbon. After this period of time, the biodegradation rate slows down due the saturation of the biomass [7]. It is possible to infer from analysis of (Fig. 1) that the biomass grows slightly with the consumption of DEK and the biomass growth decreases as the diethylketone disappears. Similar results were also observed by other authors $[11,7]$.

\subsubsection{Biodegradation of DEK by the Alternaria sp.}

The time profile of biomass concentration and the biodegradation percentage (\%) of DEK, for all the initial concentrations tested using the Alternaria sp. as biodegradation agent are shown in (Fig. 2). The biodegradation of DEK is fast in the first 160 hours, after that it slows down until values equal and higher than $95 \%$ are reached. It is possible to observe from analysis of (Fig. 2) that the biomass grows slightly with the consumption of diethylketone and decreases as the diethylketone disappears. It is therefore possible to infer that both fungi are able to efficiently biodegrade DEK, however the Penicillium sp. biodegrades the ketone faster, making it more attractive and advantageous in these processes.

Lee et al. [32] reported that the strain Penicillium sp. KT3 is capable of biodegrade small concentrations of several ketones such as DEK, methyl ethyl ketone (MEK), methyl propyl ketone (MPK), methyl isopropyl ketone (MIPK), methyl isobutyl ketone (MIBK), methyl butyl ketone (MBK). Alexieva et al. [11] reported the capacity of Trichosporon cutaneum to biodegrade mono hydroxyl derivates of phenol (resorcinol, cathecol and hydroquinone) and 2,6-dinitrophenol, $\alpha$-methylsterene and acetophenone. The ability of Fusarium solani to biodegrade MIBK was investigated by Przybulewska and Wieczorek [13]. These authors discovered that Fusarium solani was able to efficiently degrade MIBK at the rate up to $60 \mathrm{~g} \mathrm{~m}^{-3} \mathrm{~h}^{-1}$.

\subsection{Modelling of the Growth Kinetics for the Two Fungi}

Carbon substrates as ketones are usually used by microorganisms simultaneously as carbon and energy sources under controlled environmental conditions [33]. Since growth is a result of anabolic and catabolic enzymatic activities, these processes (substrate utilization or growth associated to product formation) can also be quantitatively described on the basis of growth models. The specific growth rate $(\mu)$ of a population of microorganisms and the substrate concentration $(S)$ can be related by a set of empirically derived rate laws which are considered as theoretical models. Several theoretical models such as Monod, Powell, Haldane and Luong and kinetic models were used in this study.

\subsubsection{Modelling of the Growth Kinetics of the Penicillium sp. in the Presence of DEK}

The comparison between the Penicillium sp. experimental and theoretical specific growth rate for the different kinetics models applied in this work is shown in (Fig. 3). For the Monod model the values of maximum substrate concentration $\left(C_{\max }\right)$ and of the substrate affinity constant [30] were obtained from the plot of $1 / \mu$ versus $1 / S$. The values of $\mu_{m}$ and $K_{s}$ obtained were $1.1 \times 10^{-3} \mathrm{~h}^{-1}$ and $0.808 \mathrm{~g} / \mathrm{L}$ respectively, indicating that there are no manifest signs of substrate inhibi-

Table 2. Maximum concentrations of biomass $\left(C_{m a x}\right)$, experimental specific growth rates $\left(\mu_{\text {exp }}\right)$ and correlation coefficients $\left(\mathbf{R}^{2}\right)$ obtained for the biodegradation assays with the Penicillium sp. and Alternaria sp using the method of least-squares fitting.

\begin{tabular}{|c|c|c|c|c|c|c|}
\hline \multirow{2}{*}{$\mathbf{S}_{\mathbf{0}}(\mathbf{g} / \mathbf{L})$} & \multicolumn{2}{|c|}{$\mathbf{C}_{\max }(\mathbf{g} / \mathbf{L})$} & \multicolumn{2}{c|}{$\boldsymbol{\mu}_{\text {exp }}\left(\mathbf{h}^{-1}\right)$} & \multicolumn{2}{c|}{$\mathbf{R}^{2}$} \\
\cline { 2 - 7 } & Penicillium sp. & Alternaria sp. & Penicillium sp. & Alternaria sp. & Penicillium sp. & Alternaria sp. \\
\hline \hline 0.5 & 0.520 & 1.065 & $4.0 \times 10^{-4}$ & $1.9 \times 10^{-3}$ & 0.680 & 1.000 \\
\hline 1 & 0.582 & 0.832 & $5.0 \times 10^{-4}$ & $4.0 \times 10^{-4}$ & 0.920 & 0.617 \\
\hline 2 & 0.505 & 0.873 & $9.0 \times 10^{-4}$ & $3.0 \times 10^{-4}$ & 0.992 & 0.755 \\
\hline 4 & 0.481 & 0.759 & $8.0 \times 10^{-4}$ & $6.1 \times 10^{-5}$ & 0.976 & 0.998 \\
\hline
\end{tabular}


tion for the range of concentration of DEK used, despite the insignificant growth (Table 2) and the fact that this model is not the most appropriate to describe the relation between the specific growth rate and the substrate concentration $\left(K_{s}<S_{0}\right)$. The obtained value for the coefficient of determination $\left(\mathrm{R}^{2}=0.768\right)$ corroborate that this model does not fit the experimental data properly. The experimental and predicted values for the specific growth rate at different substrate concentrations values are given in (Table 3). For the Powell model, the three kinetic constants $\left(\mu_{m}, K_{S}\right.$ and $\left.m\right)$ obtained were respectively $1.1 \times 10^{-4} \mathrm{~h}^{-1}, 0.796 \mathrm{~g} / \mathrm{L}$ and $6.3 \times 10^{-6}$. The obtained value for the coefficient of determination $\left(\mathrm{R}^{2}=0.931\right)$ showed that this model fits better the experimental data than the Monod model. (Table 3) allows the comparison between experimental data and Powell predicted data. The similarity of the results obtained for the Monod and Powell models is due to the fact that the value of maintenance rate $(\mathrm{m})$ is very small $\left(6.3 \times 10^{-6}\right)$.

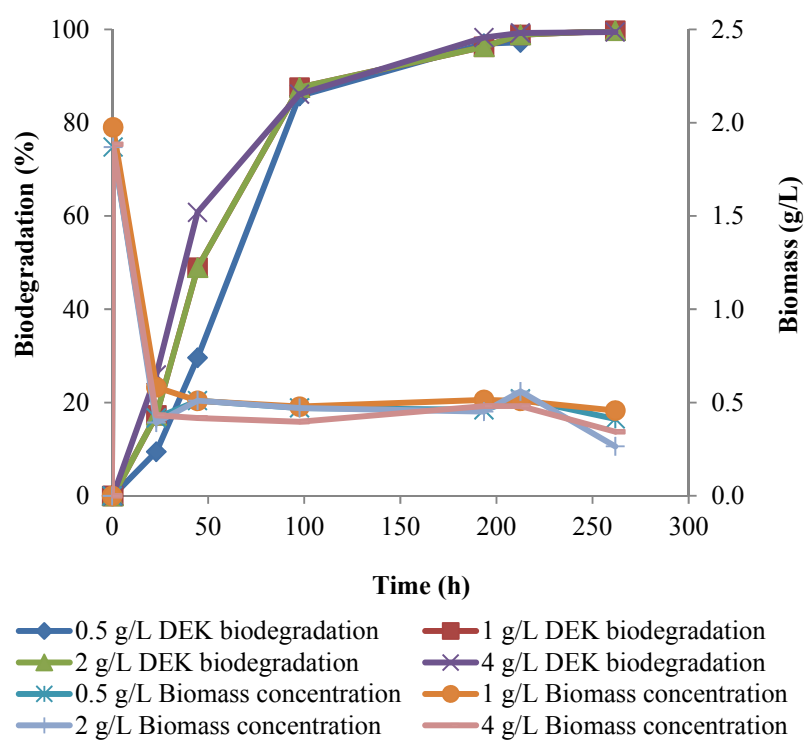

Fig. (1). Biomass concentration for the Penicillium sp. $(\mathrm{g} / \mathrm{L})$ and the biodegradation percentage (\%) as function of time, for different initial concentrations of diethylketone.

The kinetic parameters for the Haldane model $\left(\mu_{m}, K_{S}\right.$ and $K_{l}$ ) were respectively $3.5 \times 10^{-2} \mathrm{~h}^{-1}, 51.88 \mathrm{~g} / \mathrm{L}$ and 0.136 . The high value of $K s$ suggests that the biomass has a low affinity with the substrate used. The value obtained for the coefficient of determination $\left(\mathrm{R}^{2}=0.968\right)$ indicates that the predict data for the Haldane model fits better to the data compared to the Monod and Powell models. For the Luong model the kinetic parameters $\left(\mu_{m}, K_{S}, S_{m}\right.$ and $\left.n\right)$ obtained were respectively $9.6 \times 10^{-2} \mathrm{~h}^{-1}, 1229 \mathrm{~g} / \mathrm{L}, 5.943 \mathrm{~g} / \mathrm{L}$ and 0.753 , indicating the affinity of the substrate and that, above $5.943 \mathrm{~g} / \mathrm{L}$ the biodegradation and consequently the growth ceases. The coefficient of determination $\left(\mathrm{R}^{2}=0.977\right)$ showed that this model fits better the experimental data than the Haldane model.

\subsubsection{Modelling of the Growth Kinetics of the Alternaria sp. in the Presence of DEK}

For the Monod model the value for the $\mu_{m}$ and the $K_{S}$ obtained were $1.8 \times 10^{-4} \mathrm{~h}^{-1}$ and $-0.451 \mathrm{~g} / \mathrm{L}$ respectively. The small coefficient of determination $\left(\mathrm{R}^{2}=0.701\right)$ associated with the negative value obtained for the $K s$ indicates that this model does not describe the experimental data, reinforcing consequently the need and importance of testing kinetic models accounting inhibition. The experimental and predicted values for the specific growth rate at different substrate concentrations values are given in (Table $\mathbf{3}$ ).
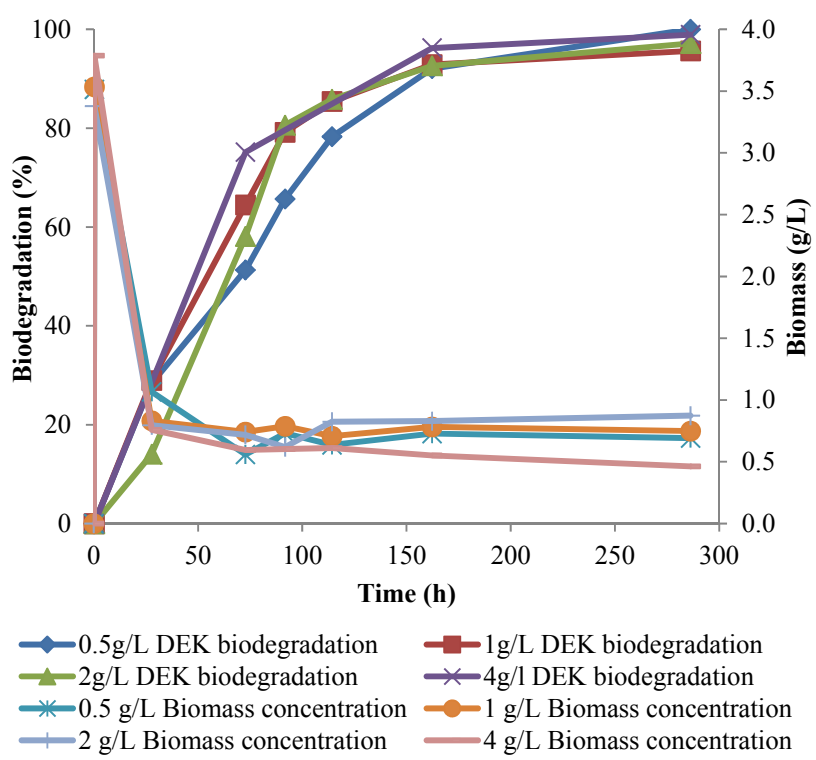

Fig. (2). Biomass concentration for the Alternaria sp. (g/L) and the biodegradation percentage (\%) as function of time, for different initial concentrations of diethylketone.

The three kinetic constants of the Powell model, $\left(\mu_{m}, K_{S}\right.$ and $m$ ) obtained were $-3.1 \times 10^{-4} \mathrm{~h}^{-1}, 4.0 \times 10^{-3} \mathrm{~g} / \mathrm{L}$ and -0.259 , respectively. The small value obtained for $K s$ and the negative values obtained for $\mu_{m}$ and $m$ showed that this model does not fit properly to the experimental data. The bad fit obtained with these two models is not unexpected as these models do not consider the inhibition effect detected during the diethylketone biodegradation process. The kinetic parameters for the Haldane model $\left(\mu_{m}, K_{S}\right.$ and $\left.K_{l}\right)$ were respectively $5.4 \times 10^{-4} \mathrm{~h}^{-1}, 0.572 \mathrm{~g} / \mathrm{L}$ and 1.163 . The high value obtained for $K_{l}$ indicates the high sensitivity of the culture towards the substrate inhibition. The value obtained for the coefficient of determination $\left(\mathrm{R}^{2}=.0 .995\right)$ associated with the obtained value for $K_{l}$ showed that this model fits properly to the experimental data. For the Luong model the kinetic parameters $\left(\mu_{m}, K_{S}, S_{m}\right.$ and $\left.n\right)$ were obtained as $2.9 \times 10^{-6} \mathrm{~h}^{-1}$, $5.092 \mathrm{~g} / \mathrm{L}, 8.016 \mathrm{~g} / \mathrm{L}$ and -3.317 , respectively. The negative value obtained for the $K s$ and $n$ showed that the experimental data is not properly described by the Luong model. The bad fit obtained for the Luong model was unexpected because this model takes into consideration the inhibitory effect, which was detected for initial concentrations of diethylketone higher than $0.5 \mathrm{~g} / \mathrm{L}$.

\subsection{Modelling of the DEK Biodegradation Kinetics by the Two Isolated Fungi}

The biodegradation kinetics is used to describe the transformation rate of organic compounds by suspended microorganism in simpler molecules and it controls the equilibrium reaching time, being therefore an important instrument for 


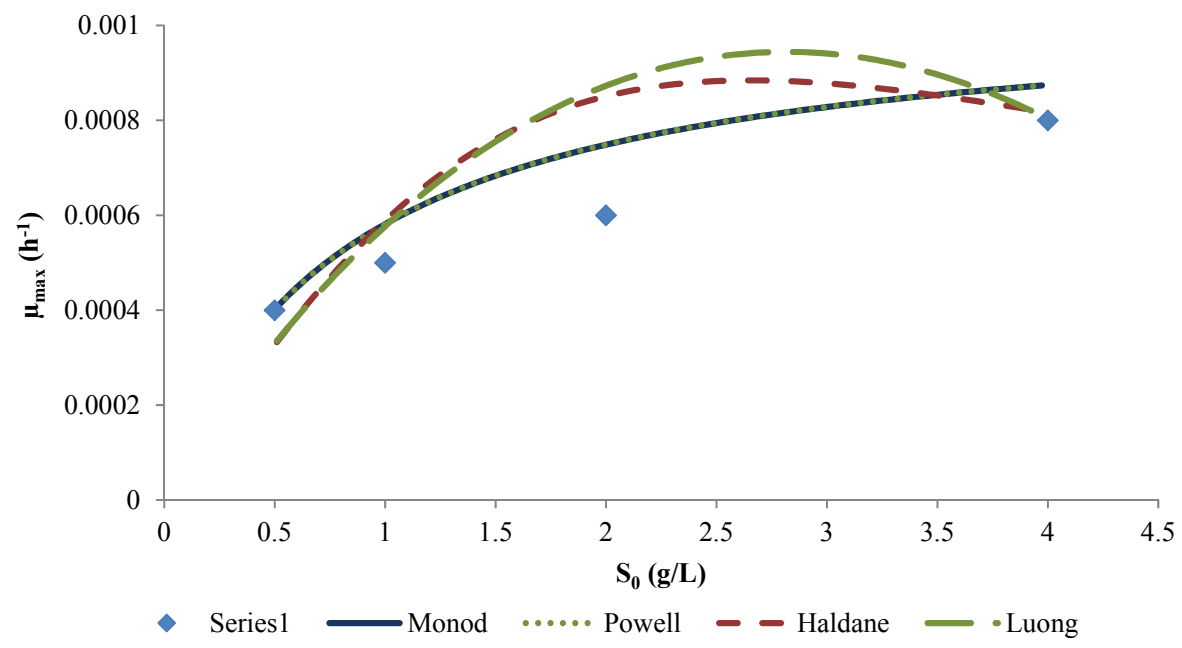

Fig. (3). Comparison between the experimental results obtained for Penicillium sp. and those predicted by the different models at different initial DEK concentrations.

Table 3. Experimental and predicted values of specific growth rate of Penicillium sp. and Alternaria sp. fungi using different growth kinetic models.

\begin{tabular}{|c|c|c|c|c|c|}
\hline$S_{0}(g / L)$ & $\boldsymbol{\mu}_{\exp }\left(\mathbf{h}^{-1}\right)$ & \multicolumn{4}{|c|}{$\mu_{\text {predicted }}\left(\mathbf{h}^{-1}\right)$} \\
\hline 0 & $-4.9 \times 10^{-4}$ & 0.0 & $-5.0 \times 10^{-4}$ & 0.0 & 0.0 \\
\hline 0.5 & $4.0 \times 10^{-4}$ & $-1.1 \times 10^{-2}$ & $-1.7 \times 10^{-2}$ & $6.1 \times 10^{-2}$ & $-1.1 \times 10^{-2}$ \\
\hline 2 & $8.7 \times 10^{-4}$ & $1.1 \times 10^{-3}$ & $1.3 \times 10^{-3}$ & $7.0 \times 10^{-4}$ & $1.1 \times 10^{-3}$ \\
\hline 4 & $7.7 \times 10^{-4}$ & $9.0 \times 10^{-4}$ & $1.0 \times 10^{-3}$ & $5.0 \times 10^{-4}$ & $9.0 \times 10^{-4}$ \\
\hline \multicolumn{6}{|c|}{ Alternaria sp. } \\
\hline$S_{0}(g / L)$ & $\mu_{\exp }\left(h^{-1}\right)$ & \multicolumn{4}{|c|}{$\mu_{\text {predicted }}\left(\mathbf{h}^{-1}\right)$} \\
\hline 0.5 & $1.9 \times 10^{-3}$ & $1.8 \times 10^{-3}$ & $3.7 \times 10^{-3}$ & 0.0 & $1.8 \times 10^{-3}$ \\
\hline 1 & $4.5 \times 10^{-4}$ & $6.0 \times 10^{-4}$ & $9.0 \times 10^{-4}$ & $5.0 \times 10^{-4}$ & $6.0 \times 10^{-4}$ \\
\hline 2 & $1.2 \times 10^{-4}$ & $5.0 \times 10^{-4}$ & $6.0 \times 10^{-4}$ & $3.0 \times 10^{-4}$ & $5.0 \times 10^{-4}$ \\
\hline 4 & $3.7 \times 10^{-4}$ & $4.0 \times 10^{-4}$ & $5.0 \times 10^{-4}$ & $2.0 \times 10^{-4}$ & $4.0 \times 10^{-4}$ \\
\hline
\end{tabular}

the process design and operational control of a biodegradation system. Zero order, pseudo-first order, pseudo-second order and three-half-order rate kinetics were used to describe the biodegradation of DEK by the Penicillium sp. and Alternaria sp. The biodegradation constants for all models were determined and are listed in (Table $\mathbf{4}$ and Table 5) for the biodegradation experiments with Penicillium sp. and Alternaria $\mathrm{sp}$, respectively.
3.4.1. Modelling of DEK Biodegradation Kinetics by the Penicillium sp.

From (Table 4) it is possible to conclude the best fit for the experimental data was obtained with the pseudo-second order model $\left(0.98 \leq \mathrm{R}^{2} \leq 1\right)$ followed by the pseudo-first order $\left(0.83 \leq R^{2} \leq 0.97\right)$, by the zero-order model $\left(0.72 \leq R^{2} \leq 0.99\right)$ and the three-half order $\left(0.68 \leq \mathrm{R}^{2} \leq 0.88\right)$. For the zero-order model the values of $S_{0}$ and of $K_{0}$ although small, increase with the increase of the initial concentration of DEK which 


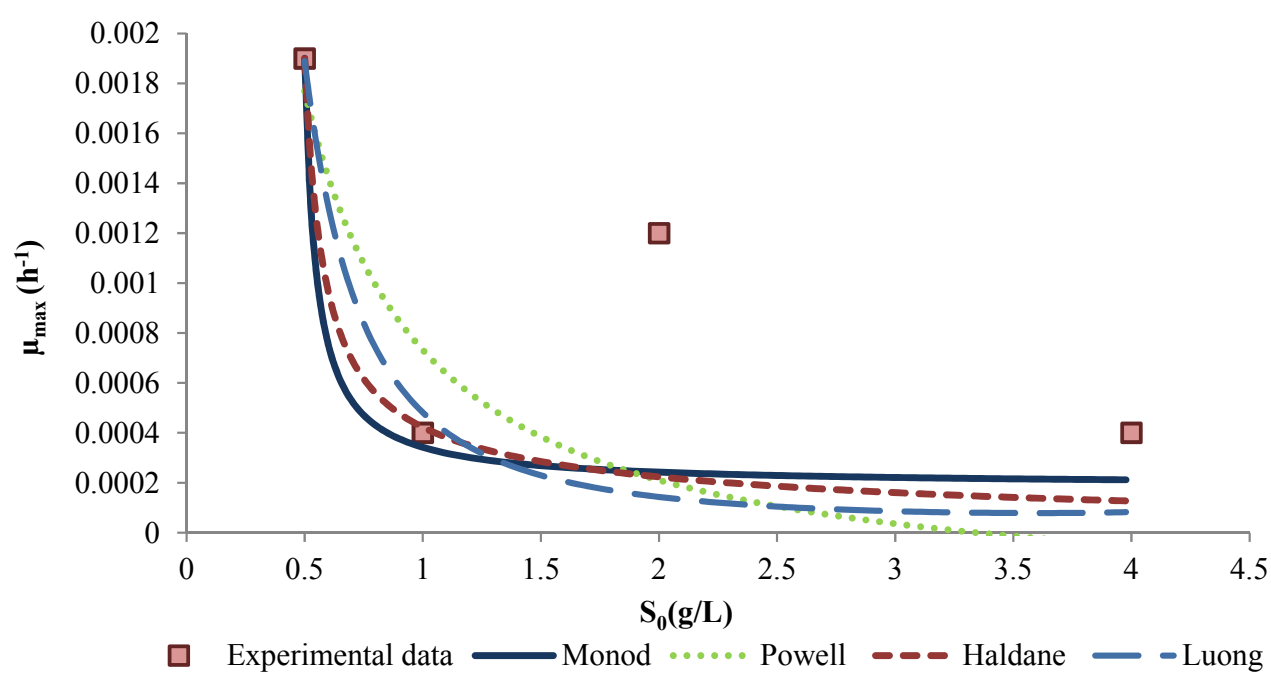

Fig. (4). Comparison between the experimental results obtained for Alternaria sp. and those predicted by the different models at different initial DEK concentrations.

Table 4. Parameters of zero-order, pseudo-first, pseudo-second and three-half-order kinetic models for different initial diethylketone concentrations and for the biodegradation with the Penicillium sp.

\begin{tabular}{|c|c|c|c|c|c|c|}
\hline$S(g / L)$ & \multicolumn{3}{|c|}{ Zero order kinetic } & \multicolumn{3}{|c|}{ Pseudo-first order kinetic } \\
\hline 0.5 & -0.072 & 0.767 & 0.960 & \multicolumn{2}{|c|}{$-6.6 \times 10^{-3}$} & 0.832 \\
\hline 1 & -0.015 & 0.525 & 0.722 & \multicolumn{2}{|c|}{$-14.1 \times 10^{-3}$} & 0.966 \\
\hline 2 & -0.020 & 2.287 & 0.983 & \multicolumn{2}{|c|}{$-9.7 \times 10^{-3}$} & 0.957 \\
\hline \multirow{2}{*}{$S(g / L)$} & \multicolumn{3}{|c|}{ Pseudo-second order kinetic } & \multicolumn{3}{|c|}{ Three-half order kinetic } \\
\hline & \multicolumn{2}{|c|}{$\mathbf{K}_{\mathbf{2}}$} & $\mathbf{R}^{2}$ & $\mathbf{K}_{31}$ & $\mathbf{K}_{32}$ & $\mathbf{R}^{2}$ \\
\hline 0.5 & \multicolumn{2}{|c|}{$1.2 \times 10^{-5}$} & 0.984 & - & - & - \\
\hline 1 & \multicolumn{2}{|c|}{$3.8 \times 10^{-5}$} & 0.996 & $9.9 \times 10^{-3}$ & $-6.0 \times 10^{-5}$ & 0.875 \\
\hline
\end{tabular}

indicates respectively, that the amount of biodegraded DEK and the rate of biodegradation increase with the raising of the initial concentration. For the pseudo-first order, the $K_{l}$ constant was found to decrease indicating that the biodegradation rate decreases with the increasing of initial concentration of DEK. In the case of the pseudo-second order model, the $K_{2}$ constant decrease with the increase of the initial concentration of DEK which suggests that the biodegradation of DEK decreases over time, which is in agreement with the obtained results. The three-half order model is not able to describe the experimental data for initial diethylketone concentrations of $0.5 \mathrm{~g} / \mathrm{L}$ and $2 \mathrm{~g} / \mathrm{L}$ which is unexpected because as previously mentioned this model considers the microbial growth during the biodegradation process. This behaviour may be explained by the reduced values of $K_{31}$ and $K_{32}$ that assume that the biodegradation rate should be extremely slow or even absent (Table 4), which was not observed.

\subsubsection{Modelling of the DEK Biodegradation Kinetics by the Alternaria sp.}

From (Table 5) it is possible to conclude that the best fit for the experimental data was obtained for the pseudosecond order model $\left(R^{2} \geq 0.99\right)$ for the biodegradations experiments conducted with an initial concentrations of diethylketone higher than $0.5 \mathrm{~g} / \mathrm{L}$, followed by the zero-order model $\left(0.84 \leq \mathrm{R}^{2} \leq 0.97\right)$. For the zero-order model, $S_{0}$ increases with the increase of the initial concentration of DEK whereas $K_{0}$ remains almost unchanged which indicates respectively that the amount of biodegraded DEK increases with the increase of initial concentration of DEK and that the rate of biodegradation remains constant. The pseudo-second order constant $K_{2}$ tends to decrease with the increase of the initial concentration of DEK suggesting that the biodegradation rate decreases over time, which is in agreement with the 
Table 5. Parameters of zero-order, pseudo-first, pseudo-second and three-half-order kinetic models for different initial diethylketone concentrations and for the biodegradation with the Penicillium sp.

\begin{tabular}{|c|c|c|c|c|c|c|}
\hline \multirow{2}{*}{$S_{0}(g / L)$} & \multicolumn{3}{|c|}{ Zero order kinetic } & \multicolumn{3}{|c|}{ Pseudo-first order kinetic } \\
\hline & $\mathbf{K}_{\mathbf{0}}$ & $\mathbf{S}_{\mathbf{0}}$ & $\mathbf{R}^{2}$ & \multicolumn{2}{|c|}{$\mathbf{K}_{1}$} & $\mathbf{R}^{2}$ \\
\hline 0.5 & -0.018 & 0.782 & 0.964 & \multicolumn{2}{|c|}{-} & - \\
\hline 1 & -0.012 & 1.562 & 0.941 & \multicolumn{2}{|c|}{-0.006} & 0.972 \\
\hline 2 & -0.012 & 1.562 & 0.941 & \multicolumn{2}{|c|}{-} & - \\
\hline 4 & -0.017 & 5.279 & 0.840 & \multicolumn{2}{|c|}{-} & - \\
\hline \multirow{2}{*}{$S_{0}(g / L)$} & \multicolumn{3}{|c|}{ Pseudo-second order kinetic } & \multicolumn{3}{|c|}{ Three-half order kinetic } \\
\hline & \multicolumn{2}{|c|}{$\mathbf{K}_{2}$} & $\mathbf{R}^{2}$ & $\mathbf{K}_{31}$ & $\mathbf{K}_{32}$ & $\mathbf{R}^{2}$ \\
\hline 0.5 & \multicolumn{2}{|c|}{$1.2 \times 10^{-5}$} & 0.754 & - & - & - \\
\hline 1 & \multicolumn{2}{|c|}{$1.0 \times 10^{-5}$} & 0.996 & 0.008 & $-4.0 \times 10^{-5}$ & 0.824 \\
\hline 2 & \multicolumn{2}{|c|}{$1.0 \times 10^{-6}$} & 0.998 & - & - & - \\
\hline 4 & \multicolumn{2}{|c|}{$1.4 \times 10^{-6}$} & 0.996 & 0.005 & $-2.0 \times 10^{-5}$ & 0.928 \\
\hline
\end{tabular}

obtained results. The pseudo-first order and the three-half order models are not able to describe the experimental data for several and different initial concentrations of diethylketone.

\section{CONCLUSION}

The obtained results showed the potential of two fungi isolated from contaminated reactor with DEK and S. equisimilis, identified as fungi belonging to the Penicillium and Alternaria genera, to efficiently biodegrade DEK in aqueous solutions (98 and 99\%, respectively). The growth kinetic model that best describe the growth of Penicillium sp. and Alternaria sp. are respectively the Luong and the Haldane model. The degradation kinetic model that best fit the results obtained for Penicillium sp. is the pseudo-second order whereas for the Alternaria sp. the pseudo-second order model only describes adequately the assays with initial concentrations of DEK higher than $0.5 \mathrm{~g} / \mathrm{L}$.

\section{CONFLICT OF INTEREST}

The author(s) confirm that this article content has no conflict of interest.

\section{ACKNOWLEDGEMENTS}

The authors would like to thank the FCT Strategic Project PEst-OE/EQB/LA0023/2013 and the Project "BioEnv Biotechnology and Bioengineering for a sustainable world", REF. NORTE-07-0124-FEDER-000048, co-funded by the Programa Operacional Regional do Norte (ON.2 - O Novo Norte), QREN, FEDER. Filomena Costa kindly thanks FCT for a $\mathrm{PhD}$ grant (SFRH/BD/77666/2011). The authors would also like to thank the help of Vânia Isabel Lopes Ferreira e Liliana Patrícia Araújo dos Santos, PhD students in the Department of Biological Engineering from the University of Minho.

\section{REFERENCES}

[1] D.P. Geoghegan, G. Hamer, M.A Deshusses, "Effects of unsteady state conditions on the biooxidation of methyl ethyl and methyl isobutyl ketone in continuous flow liquid phase cultures", Bioprocess. Eng., vol. 16, pp. 315-322, May 1997.

[2] C. Quintelas, F. Costa, T. Tavares, "Bioremoval of diethylketone by the synergistic combination of microorganisms and clays: Uptake, removal and kinetic studies", Environ. Sci. Pollut. R., vol. 20, pp. 1374-1383, June 2012.

[3] W. C. Chan, T.Y. Lai, "Interaction of compounds on biodegradation of ketone mixtures in a biofilter", J. Chem. Technol. Bio., vol. 85, pp. 416-422, March 2010.

[4] A. Datta, L. Philip, L, "Biodegradation of volatile organic compounds from paint industries", Appl. Biochem. Biotech., vol. 167 pp. 564-580, April 2012

[5] R. Hernandez, M. Zappi, J. Colucci, R. Jones, "Comparing the performance of various advanced oxidation processes for treatment of acetone contaminated water", J. Hazard. Mater., vol. 92, pp. 3350, May 2002.

[6] K-Y. Lam, D.F. Davidson, R.K Hanson, "High-Temperature Measurements of the Reactions of $\mathrm{OH}$ with a Series of Ketones: Acetone, 2-Butanone, 3-Pentanone, and 2-Pentanone", J. Phys. Chem. A., vol. 116, pp. 5549-5559, June 2012.

[7] F. Costa, C. Quintelas, T. Tavares, "Kinetics of biodegradation of diethylketone by Arthrobacter viscosus", Biodegradation, vol. 23, pp. 81-92, February 2012.

[8] S. Raghuvanshi, B.V. Babu, "Biofiltration for removal of methyl isobutyl ketone (MIBK): Experimental studies and kinetic modelling", Environ. Technol., vol. 31, pp. 29-40, January 2010.

[9] S. Raghuvanshi, B.V. Babu, "Experimental studies and kinetic modeling for removal of methyl ethyl ketone using biofiltration", Bioresource Technol., vol. 100, pp. 3855-3861, March 2009.

[10] S.N. Agathos, E. Hellin, H. Ali-Khodja, S. Deseveaux, F. Vandermesse, H. Naveau, "Gas-phase methyl ethyl ketone biodegradation in a tubular biofilm reactor: Microbiological and bioprocess aspects", Biodegradation, vol. 8, pp. 251-264, September 1997.

[11] Z. Alexieva, M. Gerginova, P. Zlateva, J. Manasiev, D. Ivanova, N. Dimova, "Monitoring of aromatic pollutants biodegradation", Biochem. Eng. J., vol. 40, pp. 233-240, December 2007.

[12] T. Essam, M.A. Amim, O.Tayeb, B. Mattiasson, B. Guieysse, "Kinetics and metabolic versatility of highly tolerant phenol degrading Alcaligenes strain TW1”, J. Hazard. Mater., vol. 173, pp. 783-788, January 2010. 
[13] K. Przybulewska, A. Wieczorek, "Biodegradation of methyl isobutyl ketone (MIBK) by Fusarium solani", Arch. Environ. Prot., vol. 35, pp. 3-10, 2009.

[14] S. Raghuvanshi, B.V Babu, "Biodegradation kinetics of methyl isobutyl ketone by acclimated mixed culture", Biodegradation, vol. 21, pp. 31-42, June 2009.

[15] S. Vijayaraghavan, T. Srinivasaraghavan, S., Musti, S., Kar, T. Swaminathan, A. Baradarajan, "Biodegradation of phenol by arthrobacter and modelling of the kinetics", Bioprocess. Eng., vol. 12, pp. 227-229, 1995.

[16] L.L- Zhang, R.Y. Zhu, J.M. Chen, W.M. Cai, "Biodegradation of methyl tert-butyl ether as a sole carbon source by aerobic granules cultivated in a sequencing batch reactor", Bioproc. Biosyst. Eng., vol. 31 , pp. 527-534, October 2008

[17] H.I. Atagana, "Biodegradation of phenol, o-cresol, m-cresol and pcresol by indigenous soil fungi in soil contaminated with creosote", World J. Microbiol. Biotechnol., vol. 20, pp. 851-858, April 2004.

[18] J.R. Haas, E.H. Bailey, O.W. Purvis, "Bioaccumulation of metals by lichens: Uptake of aqueous uranium by Peltigera membranacea as a function of time and pH", Am. Mineral., vol. 83, pp. 14941502, 1998 .

[19] D.Y.Kim, Y.H. Rhee, "Biodegradation of microbial and synthetic polyesters by fungi", Appl. Microbiol. Biot.., vol. 91, pp. 300-308, January 2003.

[20] B. Volesky, Z.R. Holan, "Biosorption of heavy metals", Biotechnol. Progr., vol. 11, pp. 235-250, May 1995.

[21] M. Ayala, M.A. Pickard, R. Vazquez-Duhalt, "Fungal enzymes for environmental purposes, a molecular biology challenge", J. Mol. Microbiol. Biotechnol., vol. 15, pp. 172-180, August 2008.

[22] W. Chai, Y. Handa, M. Suzuki, M. Saito, N. Kato, C.A. Horiuchi, "Biodegradation of bisphenol A by fungi", Appl. Biochem. Biotech., vol. 120, pp. 175-182, March 2005.

[23] M. Pipíška, M. Horník, L.U., Vrtoch, J. Augustín, J. Lesný, "Biosorption of $\mathrm{Co}^{2+}$ ions by lichen Hypogymnia physodes from aqueous solutions", Biologia, vol. 62, pp. 276-282, June 2007.
[24] J. Monod, "The growth of bacterial cultures", Annu. Rev. Microbiol., vol. 3, pp. 371-394, October 1949.

[25] E.O. Powell, "The growth rate of microorganisms as function of substrate concentration", in Microbial Physiology and Continuous Culture, Proceediings of the 3rd International Symposium on Microbial Physiology and Continuous Culture. Her Majesty's Stationery Office, London. pp. 34-56.

[26] J.F. Andrews, "A mathematical model for the continuous culture of microorganisms utilizing inhibitory substance", Biotechnol. Bioeng., vol. 10, pp. 707-723, 1968.

[27] J.H.T. Luong, "Generalization of monod kinetics for analysis of growth data with substrate inhibition", Biotechnol. Bioeng., vol. 29, pp. 242-248, February 1987.

[28] V.H. Edwards, "The influence of high substrate concentrations on microbial kinetics", Biotechnol. Bioeng., vol. 12, pp. 679-712, September 1970

[29] M. Khamis, F. Jumean, N. Abdo, "Speciation and removal of chromium from aqueous solution by white, yellow and red UAE sand", J. Hazard. Mater., vol. 169, pp. 948-952, September 2009.

[30] P. Saravanan, K. Pakshirajan, P. Saha, "Batch growth kinetics of an indigenous mixed microbial culture utilizing $\mathrm{m}$-cresol as the sole carbon source", J. Hazard. Mater., vol. 162, pp. 476-481, February 2009.

[31] W. Brunner, D.D. Focht, "Deterministic three-half-order kinetic model for microbial degradation of added carbon substrates in soil", Appl. Environ. Microb., vol. 47, pp. 167-172, January 1984.

[32] T.H. Lee, J. Kim, M-J. Kim, H.W. Ryo, K-S. Cho, "Degradation characteristics of methyl ethyl ketone by Pseudomonas sp. KT-3 in liquid culture and biofilter", Chemosphere, vol. 63, pp. 315-322, April 2006.

[33] C. Quintelas, B. Fonseca, B. Silva, H. Figueiredo, T. Tavares, "Treatment of chromium(VI) solutions in a pilot-scale bioreactor through a biofilm of Arthrobacter viscosus supported on GAC", Bioresource Technol., vol. 100, pp. 220-226., January 2009. 